17 Thorlby R, Smith J, Williams S, Dayan M. The Francis Report: one year on. London: Nuffield Trust, 2014. Available online at www.nuffieldtrust.org.uk/sites/files/nuffield/publication/140206_the_ francis_inquiry.pdf [Accessed 10 July 2015].

18 Point of Care Foundation. Staff care: how to engage staff in the NHS and why it matters. London: Point of Care Foundation, 2014. Available online at www.pointofcarefoundation.org.uk/ Downloads/Staff-Report-2014.pdf [Accessed 27 August 2015].

19 Wheatley MJ. Leadership and the new science: discovering order in a chaotic world. San Francisco, CA: Berrett-Koehler, 2006.

20 The King's Fund. (2014). Culture and leadership in the NHS: the King's Fund 2014 survey. London: King's Fund, 2014. Available online at www.kingsfund.org.uk/sites/files/kf/field/field_ publication_file/survey-culture-leadership-nhs-may2014.pdf [Accessed 10 July 2015].
21 Health Foundation. Francis Inquiry - one year on. London: Health Foundation, 6 February 2014. Available online at www.health.org. uk/news-and-events/press/francis-inquiry-one-year-on/ [Accessed 10 July 2015].

22 West M, Eckert R, Steward K, Pasmore B. Developing collective leadership for health care. London: King's Fund, 2014.

23 Bridges L. Mid Staffs: what impact has it had? London: Ipsos Mori May, 2015. Available online at www.ipsos-mori.com/newsevents/ blogs/makingsenseofsociety/1675/Mid-Staffs-what-impact-hasit-had.aspx [Accessed 10 July 2015].

Address for correspondence: Ms K Lynas, 3 The Embankment, Sovereign Street, Leeds LS1 4GP, UK.

Email: karen.lynas@leadershipacademy.nhs.uk

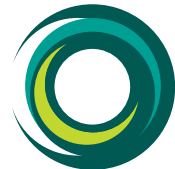

\title{
Faculty of Medical Leadership and Management
}

\section{Professionalising medical leadership}

\author{
Author: Peter Lees ${ }^{\mathrm{A}}$
}

There is a growing body of evidence linking leadership and patient outcome including mortality. There is also evidence demonstrating the importance of medical leadership to the delivery of high-quality care at an organisational level. This contrasts with a lack of structure for medical leadership careers or medical leadership development and, until recently, the lack of standards to guide individuals and organisations. The UK medical royal colleges and the Academy of Medical Royal Colleges began to address this by the establishment of the new Faculty of Medical Leadership and Management in 2011. The first standards are now published and a range of other activities aimed at improving medical leadership are underway. In parallel with this professionalising of medical leadership, the profession needs to recognise its value, encourage skills development alongside clinical training and play its role in leadership at all levels to leverage the maximum benefits for patients.

KEYWORDS: Medical leadership, management, standards, professionalism, clinical outcome

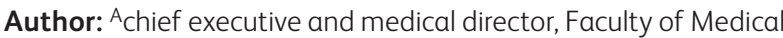
Leadership and Management, London, UK

\section{Introduction}

Leadership is a prime determinant of organisational performance across all sectors. Many industries focus heavily on succession planning and the recruitment and development of good leaders. They devote significant attention to the nurturing and development of leadership talent - all to secure enhanced performance.

Healthcare is not exempt from the benefits of good leadership and high-performing teams; indeed, in health, there is the added, poignant dimension that associated quality metrics include mortality and morbidity.

\section{Leadership in healthcare - the evidence}

A recent literature review of leadership in healthcare ${ }^{1}$ pointed to a relative dearth of high-quality research into healthcare leadership, despite its potential to do good and ill! There is, however, a growing body of evidence linking leadership and teamwork with clinical outcomes including mortality. ${ }^{2}$ Despite this critical association and the encouraging investment in leadership development by NHS organisations in recent years, there is little evidence to guide the appropriate design of interventions. There is also limited knowledge regarding value for money from a wide range of popular leadership development approaches.

While much of the leadership and management research relating to clinical outcomes is not profession specific there 
is relatively new evidence pointing to the value doctors bring. In 2011, Goodall showed that in the USA, the presence of a physician chief executive, as opposed to a professional manager, was shown to be associated with better hospital performance, whereby physician-led hospitals achieved $25 \%$ higher quality scores. ${ }^{3}$ This increase did not apply to leaders from other clinical backgrounds. Veronesi et al found that UK trusts with a higher proportion of doctors on the board performed better. ${ }^{4}$ This also did not apply to other clinical professionals. Furthermore, the UK Medical Engagement Scale demonstrates an association between Care Quality Commission organisational quality ratings and the engagement of doctors. ${ }^{5}$

These findings raise significant issues given the current state and status of medical leadership. There is a widespread lack of enthusiasm among doctors to take on leadership roles when compared and contrasted with academia, education or pure clinical medicine. This is not just a British phenomenon and afflicts countries as far afield as the USA, Canada, South Africa and Australia, as discussed at the recent World Federation of Medical Managers 2015 Meeting (personal communication). The reasons will surprise few and have been surveyed many times, most recently by the Canadian Association of Physician Executives. ${ }^{6}$ High on the list is the negative attitude towards medical leadership and management within the profession with, for example, much greater status afforded to equivalent academic roles. Frustrating to the converted, this is perhaps unsurprising given that leadership and management are not yet subject to the same exacting criteria as clinical medicine. There have, until recently, been no defined standards of medical leadership, minimal focus has been placed on training and there are no specific postgraduate qualifications. Many medical leaders and managers have undoubtedly made a huge contribution over many years but there are no measures or benchmarks to demonstrate this.

Recent decades have been defined too much by very public failures in the NHS. All have begged the question: Where was the medical leadership? Depressingly, in the 2015 report of the Morecambe Bay investigation, ${ }^{7}$ a medical leader is quoted as saying:

But if you work with another three consultants who will not undertake any responsibility [for tackling problems] then there is a limit to what you can do. You can sit down with them. You can mention it to the Medical Director. You can mention it to the Chief Executive.

This poses deeply uncomfortable questions. How can the profession tolerate a system where its operational leaders can be so ill-equipped and when the end result is so clear to see? It would be deemed completely unacceptable were this an equivalent issue of clinical competence.

\section{Standards of medical leadership and management}

UK medicine is rightly proud of the standards which underpin clinical practice. Logically, it must apply the same rigour to leadership and management if healthcare is to capitalise on the consequent benefits to patients and organisations. To this end, in 2011 the Academy of Medical Royal Colleges and its constituent bodies established the Faculty of Medical Leadership and Management (FMLM). This was commended in the 2013 Report of the Mid Staffordshire NHS Foundation Trust public inquiry 8 against the backdrop of a key recommendation: 'there is little doubt that enhancement of the status of healthcare management and leadership as a profession is sorely needed'. It is sobering in the flurry of activity which has followed the Francis report that only the medical profession has responded in full to this recommendation.

FMLM published the first UK Leadership and management standards for medical professionals ${ }^{9}$ in 2015. The links to appraisal and revalidation of medical leaders have been defined, and the process of accreditation is being designed. Individuals and organisations now have a benchmark to aid assessment, evaluate progress and inform the appointments process. This is an important foundation to systematically address the need for high-calibre medical leaders at all levels of the system.

\section{Medical leadership as a career}

Historically, medical leaders have attained formal roles through their own initiative, hearing about potential jobs has been haphazard, internal appointments are the norm and competition is often sparse. There is no guidance for appointment processes and, unlike consultant interviews, there may be no external scrutiny. Job descriptions are frequently little more than lists which have been added to over time and the infrastructure support varies hugely with some jobs quite simply set up to fail.

Aspiring medical leaders frequently seek advice regarding appropriate and available development opportunities and the notion of 'doing an MBA' is often raised. For some this will be excellent and some MBAs are very suitable but for most, the combination of cost, time commitment and appropriateness of available programmes does not make this a viable or wise option. With or without an MBA, leadership remains fundamentally about doing: getting results, with and through people! Not surprisingly therefore, the evidence regarding the impact of classroom learning is limited. Leadership is also contextual which offers a further challenge to popular leadership programmes. Development ideally needs to be as close to the place of work as possible to gain relevance and be maximally effective. ${ }^{9}$

FMLM is addressing all of these issues. What remains to be tackled and requires broader support is the negativity within the profession towards medical leadership and management. National bodies and, in particular the medical royal colleges, have a major responsibility here. Professionalising medical leadership may help to raise quality and to earn respect. Also, those currently in leadership roles have an advocacy rolereferences to 'going over to the dark side' are deeply unhelpful, but medical leaders use the term liberally too which does nothing to help the status of complex and challenging roles.

\section{The tools to do the job}

Medical leaders must also fight for appropriate resources to allow them to succeed. Medical leadership is far too important to be relegated to being spare-time activities if indeed, spare time exists anymore! Equally, infrastructure support needs to be commensurate with the size of the task and it is a challenge for leaders not to be complicit through accepting roles which cannot be discharged well for lack of appropriate resources. 
Of course, leadership is not the sole province of those with it in their title. Wherever there is a team endeavor, ie most if not all of the time in clinical medicine, there is leadership. Here the association with patient outcome is probably more direct than in the more senior titled roles. The foundation year doctor finding herself the first medically qualified person at a clinical emergency needs leadership and management skills every bit as much as she needs clinical skills if the team is to function in the best interests of the unfortunate patient. In short, all doctors need a level of leadership and management skills leading to the inescapable conclusion that learning should begin early and many would argue in medical schools. That learning needs to be supported throughout the training years - a fact not lost on over 1,000 trainees and students who have joined FMLM.

\section{Conclusions}

To conclude, it is time to take medical leadership seriously, time for it to move from amateur sport to a highly professional one. The newly defined standards of medical leadership need to be adopted swiftly and be adopted as the basis for appointing and monitoring the progress of medical leaders. There needs to be a relentless, systematic drive to identify, support and develop good medical leaders. Individuals have a responsibility to apply the same standards to their leadership career as they do to their clinical career, to be strong advocates for medical leadership, and to spot and nurture up-and-coming talent. Significant progress has been made in recent years and further success will be measured in improved quality of patient care.

\section{References}

1 West M, Armit K, Loewenthal L et al. Leadership and leadership development in healthcare: the evidence base. London: FMLM, 2015.

2 West MA, Borrill C, Dawson, J et al. The link between the management of employees and patient mortality in acute hospitals. Int $J$ Hum Resour Manag 2002;13:1299-310.

3 Goodall AH. Physician-leaders and hospital performance: Is there an association? Soc Sci Med 2011;73:535-9.

4 Veronesi G, Kirkpatrick I, Vallascas F. Clinicians on the board: what difference does it make? Soc Sci Med 2013;77:147-55.

5 Spurgeon P, Barwell F, Mazelan P. Developing a medical engagement scale (MES). Int J Clin Leadersh 2008;16:213-23.

6 Van Aerde J. What do physicians need to lead? Can J Physician Leadersh 2015:1:3-7.

7 Kirkup B. The report of the Morecambe Bay investigation. London: Stationery Office, 2015. Available online at www.gov.uk/government/ uploads/system/uploads/attachment_data/file/408480/47487_MBI_ Accessible_v0.1.pdf [Accessed 10 July 2015].

8 Report of the Mid Staffordshire NHS Foundation Trust Public Inquiry. Chaired by Robert Francis QC. London: Stationery Office, 2013. Available online at www.midstaffspublicinquiry.com/report [Accessed 10 July 2015].

9 Faculty of Medical Leadership and Management. Leadership and management standards for medical professionals. London: FMLM, 2014. Available online at www.fmlm.ac.uk/professional-development/ accreditation-and-standards/the-leadership-and-management-standards-for [Accessed 10 July 2015].

Address for correspondence: Mr P Lees, Faculty of Medical Leadership and Management, 2nd Floor, 6 St Andrews Place, London NW1 4LB, UK.

Email: peter.lees@fmlm.ac.uk 